\title{
Technology Innovation To Modern Designs Gasing or Top Spinning in Community Culture
}

\author{
Azlina Musa
}

\begin{abstract}
This paper will discuss the technology that brings innovation to modern design in the culture of society. The design of modern technology has helped reintroduce old games that are gasing or top spinning to the new generation. The invention of modern technology in the redesign of the lace design has helped to bring the interest of today's generation closer to the lace game. Modern technology in the design of the original design has also gained popularity among people of all ages. In this regard, how the extent to which modern technology plays a role in influencing the production of a variety of design in gasing or top spinning the needs and requirements of modern society. In addition, why the extent to which the game of gasing or top spinning can help create team spirit in the game of gasing or top spinning. In addition to examining how modern technologies that use new technologies have been implementing the reforms in the field are gaining popularity among the community culture. Re-documenting this study could add further sources of reference to other researchers in the future.
\end{abstract}

Keywords : technology, gasing or top spinning, efficiency, culture

\section{INTRODUCTION}

Rapid advances in technology play a key role in helping to broaden the game play. The influence that technology has had is so powerful that it can give birth to new form of play that looks to meet the needs of society. In addition to the traditional game with the application of technology elements brought into the game gasing or top spinning of lore that was once unappreciated, modern day modernization has become very popular.

In this regard, the tradition of old fashioned games of traditional and modern gasing or top spinning society has been redesigned in line with the advancement of modern technology. The new design of the original is a substitute for the traditionally worn out and cannot meet the needs of today's society. Weaknesses in the old design require technology for the improvement of the original to gasing or top spinning the needs of modern society.

In this regard, the new design of the new technology with the latest technology in the design of modern design is well accepted by the public. In fact, the community's interest in

Revised Manuscript Received on April 25, 2020.

* Correspondence Author

Azlina Musa*, Pusat Pendidikan Asas dan Liberal, Universiti Malaysia Terengganu, 21030 Kuala Nerus, Terengganu, Malaysia

(C) The Authors. Published by Blue Eyes Intelligence Engineering and Sciences Publication (BEIESP). This is an open access article under the CC BY-NC-ND license (http://creativecommons.org/licenses/by-nc-nd/4.0/) modern design is pervasive and growing among the people living in the village or the urban community. However modern technology in line with technology is gaining popularity among people of all ages who love to play with modern games gasing or top spinning.Old fashioned gasing or top spinning are a bit difficult to play because the gasing or top spinning is an object that is rotated by a rope or even more precisely around the rope. Old games gasing or top spinning are traditionally played to make up time that can only be played in outdoor play areas. The old way of playing is to position the left leg truss in front of the axle and lower it to the back of the shoulder and move it to the next turn. The design of modern technology is still much to be done in an effort to keep the old game gasing or top spinning of good will out of the ordinary and to be enjoyed by the people. One of the technological advances of modern technology is to revise the old. Among the new features is that through the beyblade, the polymer gasing or top spinning can help enhance the laminate game as well as add value to the physical design of the lining that is able to attract the public to enjoy the gasing or top spinning.In addition, technological advances have also introduced gaming applications that are also playable through gaming applications that are also playable through gaming applications either by playing on a mobile phone or computer game gasing or top spinning. Technology has introduced gaming on mobile or computer applications that are accessible at the fingertips only. Among the games that can be accessed on a mobile phone or computer are such games gasing or top spinning that have their own names to capture the interest of the community such as beyblade burst rivals, knives carsh, gyro buster, blade battle and more play games gasing or top spinning. Each computer game has its own unique features that are able to connect the community to the game of computing. Technological innovations in the game gasing or top spinning of play can be played anytime at home or in the community. In addition, modern gadgets are easily accessible on mobile or computer games are becoming more popular and gaining popularity among the public than the old ones. Computerized gaming is the only way home games can be played. In addition, modern technology is also in the process of introducing beyblade design, polymer coating is helping to regain the dignity of the traditional games gasing or top spinning in line with the high tech age. The modern design of the case is to help the community to feel the game for themselves whether on a mobile phone or computer. In addition, the new design of the handles makes it easier for the community to play real life home games. In this regard,

Published By:

Blue Eyes Intelligence Engineering \& Sciences Publication 


\section{Technology Innovation To Modern Designs Gasing or Top Spinning in Community Culture}

beyblade or polymer coating allows the community to play real life in order to fill in their free time at home or outdoors.

In addition, high end gaming technology is one of the ways in which people of all ages are interested in recognizing games gasing or top spinning as one of the most traditional games of the time.

\section{RESULT AND DISCUSSION INNOVATIONS GASING OR TOP SPINNING IN MODERN TECHNOLOGY}

Skating games are spreading all over the world and including our region. The game of gasing or top spinning is believed to have been played for the last three and a half centuries (Ghazali Dato Yusoff, 1989). Landing games are a bit far behind than other traditional games. However, modern technology has helped bring innovation to the game of play through an exterior design that is capable of reaching the public's response to the game. Beginners in the manufacture of gasing or top spinning designs are tin and wood laminates played in all states of Malaysian. Gasing or top spinning made tin cans are very heavy and difficult for the public to play gasing or top spinning. However the advances in modern technology have led to changes in the stylish, lighter and more sophisticated designs of the people who love the game. Apart from that, the paintings are also provided with a variety of colorful patterns and colors that have the potential to appeal to the community. However, modern technology has brought a change to the variety of designs and the public has a preference for such games. However, the shape and manner of the game of play can play the same way that the play of the same game creates unity and team spirit in every match. So the similarity between the old and the modern is that the way the game is still maintained in each games is to determine whether to lose or win in the game gasing or top spinning.

Gasing or top spinning games are not only played by adults but also by youth and children. The play gasing or top spinning is not only an adult game but it is also played by children, teenagers and the elderly, but also enjoys playing games for free time. Their ability to play catch-up for teamwork, efficiency, speed and teamwork. In addition, they can also strengthen their relationships. In addition, this game of play can also build friendships between them. Besides, this is not a new game but it was played before the war of the world. Today, the original is being reintroduced by today's generation through the technological innovations introduced by the old. New technologies are being absorbed in modern game design gasing or top spinning. The play is not only played by adults but also by teenagers and children. This game of play is played to fill your free time. The attractive and beautiful gasing or top spinning of the tin have begun to receive a favorable response in ancient times. Gasing or top spinning games are not enjoyed by people of all ages, from children to adults, they are interested in such games.

\section{A. Method game gasing or top spinning}

The old fashioned way of playing games is generally the same as turning, pushing and even defending. The only difference is the way the play is played. Among them are the talar tops, plate tops and expandable tops found in the state of Johor. In addition, gasing or top spinning tin foil was popular

Retrieval Number: D7792049420/2020@BEIESP

DOI: 10.35940/ijeat.D7792.049420

Journal Website: www.ijeat.org in Kelantan in ancient times. While in the state of Selangor, it is also known for its steel mills, sea slabs and waterproofing. Meanwhile, in Kelantan it is known as the gasing or top spinning as the tin, the foam and the state of Kedah are also known for the nail. For the states of Sabah and Sarawak, there are different forms of luggage called lundu, gianne, borneo gown and brunai. While playing this game is not very different from one another. The only difference between them is their shape and size. Some of the tools needed in the old game are quite complicated and difficult to play include the gear, the rope, the smoker, the puddle, the pole, the bong, the rubber band and the little oil. However, modern technology brings the tools that are needed in the game of simplicity and is simple to play. In this regard, technology through modern design helps to make the game more accessible to the public.

In addition, in the old days, the game of gasing or top spinning is that it covers a lot of energy and it usually does not turn the lid itself. This is intended to save energy on the rotator casing because rotating, the spinner would tie the end of the rope to his wrist. For the rotator to rotate the handle with the right hand and the fork is rotated clockwise. Instead the rotator will rotate with the left hand, the handle must be rotated clockwise. In addition, a well-rounded and experienced turner can turn well. The spine that he twists is also rarely related. An experienced spinner is also able to estimate the ideal positioning distance between himself and the barrel. Usually the distance between the turn is too close or too close to the bong so the tire will not fall or rotate over the bong. In addition, someone who wants to rotate the pedal should also first learn the technique of rotating the pedal and after that he must practice hard. It is not only the distance that you need to be aware of but the technique of throwing the throttle and also pulling the strings must be learned and known. The technique of throwing and pulling ropes is a combination of the skills and skills needed in the game of lacquer. While rotating the handle, the spinner extends the blade slightly forward with both hands. Then, with the other hand, the pedal will swing backwards. Next, the spinner throws the beam toward the bar. Then the strap that was already fastened to the wrist was pulled back quickly. This is where the experience and knowledge of the cutting-edge technique comes from. If the rope is pulled slowly, it will fall out of the bong and if it is too fast it will be connected. Therefore, rotating the joints with. The ligands is not only dependents on the strong physical force as there is a strong rotating force but is not able to rotate with the ligaments. On the other hand, there is less physical rotation but it can rotate with the ligament. This shows that the technical skills and experience of playing the gasing or top spinning are essential in the match.In addition, during the game where the spinner was about to turn around, fans and supporters of both teams would be around the playing field. They want to see and give moral support to their respective players. Pushing and clapping are not allowed during the rotation. This is to avoid any misunderstanding between the two.

Published By:

Blue Eyes Intelligence Engineering

\& Sciences Publication

(C) Copyright: All rights reserved. 
While the way of the game of gasing or top spinning is so challenging that it is not only physically challenging but it also involves the sharpness of mind to find the best strategy for winning the game of gasing or top spinning. As well as when the vase is being tucked away the audience will circle around the game bar to see the game gasing or top spinning. When the stage is about to lose, more and more people will be cheering. They will each make predictions about the losing goal first and the hopeful winning. Such is the case with every style in the match. In addition to modern games, it is a game that does not require complicated or expensive equipment. Modern play equipment is free of cost. The old lawn requires that the yard is ground and usually only in the yard. However, technology has introduced modern gaming gasing or top spinning that can only be played on smartphone applications or home computers. This helps ease the game of play by the public. But the old form of play and the modern one are the same as the match for deciding to lose or winning the match.

\section{B. Modern Technology Brings The Revamp Of The Old Design To The Modern Design That Is Of Interest To Recognize The Original Games In The Culture Of The People}

Through modernization the social, economic and game structures have changed (Mohd Taib Osman and Wan Kadir Yusoff, 1989). Accordingly, at the beginning of the timber was a design gasing or top spinning made of tin, wood such as cherry wood, trunks, chisel and lumber. Gasing or top spinning made of tin and wood trim are durable and relatively inexpensive and the price of gasing or top spinning made of tin and wood trim is more expensive. However, modern technology has brought about the revival of old design through polymer design and beyblade. Polymers are more durable, uniform, safety and inexpensive. Thus, polymer coating and beyblade coating is a modern technology that brings innovation to existing design. However, the styles and methods of traditional games and modern technology games gasing or top spinning are mutually exclusive.Traditional games gasing or top spinning of identity are those that identify with a particular race or ethnicity and can be regarded as a platform of unity and unity and self-esteem. Gasing or top spinning is a traditional game that should be preserved. Gasing or top spinning is a traditional Malay game played during free time. However, the game of gasing or top spinning has been transformed with modern technology in cutting-edge games is to transform wood-based materials into materials made of technologically advanced materials so that durable design is more durable. Naturally, the gasing or top spinning is game that is always known as a wooden tool that includes the new dung, rambai, buffalo, tramp and so on. The rope straps are made from the bark of the tree and are now turning into nylon. However, modern technology has now brought about the modernization of plastic, steel for a variety of reasons, which is to last longer and to prevent continued dependence on the tree. Modern technology in the design of the lace has made it even more widely accepted internationally in all countries. Without the reliance on modern technology the long-standing tradition of game play will gradually diminish and the position of the original will be lost by itself without the efforts of modern technology to develop it. In addition, the weakened interest in traditional folklore has led to a lack of public response to tradition. In addition, modern technology that features modern design in the gasing or top spinning of games can attract many people to recognize modern design. Therefore, emerging technologies are tailored to the needs and wants of the market. The old style is beginning to adapt to the demands of modern technology. The everchanging technology of modern technology is that the position of the old design may change from the original design of the old. The next generation is having a hard time getting to known the old one. This is because the fabric created for the market no longer emphasizes the traditional properties of its shape and function. Modern gadgets available in the current market requirements. The possibility of modern customized by incorporating modern elements and leaving traditional elements in the old style. The cultural elements of the tradition are also placed in difference contexts and functions and are certainly new things. Such developments if left to a large extent, the cultural elements of tradition are trying to be popular in contexts and positions that are different from where they came from. The advances in modern technology have led to today's young people becoming increasingly separated from traditional cultural elements. The younger generation today is more exposed to modern design. This effect can also reduce their interest in the cultural heritage itself. They are also less exposed to the cultural elements of tradition that help to reinforce their awareness of the existence of the canvas.

\section{METHODOLOGY}

The methodology findings are the result of observations based on detailed description of technology innovation to modern design gasing or top spinning in community culture. In addition to the results of the through direct quotations from statement about the acceptance of the technology innovation to modern design gasing or top spinning within the community culture.

\section{CONCLUSION}

Modern technology helps to bring the game gasing or top spinning old design to the modern design in line with the times. Indeed modern design with the application of modern technology in the design of old design is the ability to restore the old style of interest to the new generation today.

\section{REFERENCES}

1. Ghazali Dato Yusoff. 1989. Pengantar Kesenian Kelantan. Kuala Lumpur: Perpustakaan Universiti Malaya.

2. Mohd Taib Osman \& Wan Kadir Yusoff. 1986. Kajian budaya dan masyarakat di Malaysia. Kuala Lumpur: Dewan Bahasa dan Pustaka.

\section{AUTHORS PROFILE}

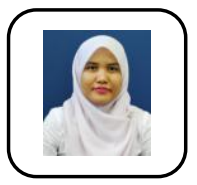

Azlina Musa, Studies Pusat Pendidikan Asas dan Liberal, Universiti Malaysia Terengganu

Published By:

Blue Eyes Intelligence Engineering

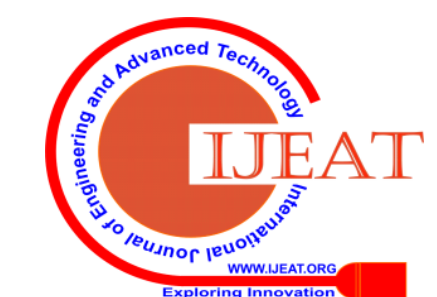

